\title{
Upregulation of liver kinase B1 predicts poor prognosis in hepatocellular carcinoma
}

\author{
XIAOLONG TAN, ZHIBIN LIAO, HUIFANG LIANG, XIAOPING CHEN, \\ BIXIANG ZHANG and LIANG CHU \\ Hepatic Surgery Center, Tongji Hospital, Tongji Medical College, \\ Huazhong University of Science and Technology, Wuhan, Hubei 430030, P.R. China
}

Received April 3, 2018; Accepted August 3, 2018

DOI: 10.3892/ijo.2018.4556

\begin{abstract}
The majority of studies report that liver kinase B1 (LKB1) acts as a tumor suppressor by inhibiting cell proliferation and metastasis. The present study investigated the expression pattern of LKB1 in 2 cohorts of paired hepatocellular carcinoma (HCC) and analogous non-cancerous tissues (ANT). The results indicated that LKB1 was upregulated in HCC vs. ANT tissues, and that high expression of LKB1 was associated with a higher number of tumor foci, larger tumor size, poorer tumor differentiation, EdmondsonSteiner grade, Barcelona Clinic Liver Cancer grade and tumor-node-metastasis stage. Furthermore, high LKB1 expression was associated with poor overall survival (OS), shorter disease-free survival and early recurrence. Univariate and multivariate analyses demonstrated that high LKB1 expression may serve as an independent prognostic marker for OS, but not for recurrence. In addition, knockdown of LKB1 expression in HCC cell lines inhibited cell proliferation and subcutaneous tumor growth by promoting cell apoptosis. Therefore, the findings of the present study suggest a protooncogenic role of LKB1 in HCC.
\end{abstract}

\section{Introduction}

Liver kinase B1 (LKB1), also referred to as serine threonine kinase 11, plays diverse roles in cellular proliferation, energy metabolism, apoptosis and polarity, by regulating a variety of substrates. LKB1 activates at least 14 adenosine monophosphate (AMP)-activated protein kinase (AMPK)-associated kinases, and the most extensively investigated substrate is AMPK (1). LKB1/AMPK is activated when the AMP/ATP

Correspondence to: Dr Liang Chu or Professor Bixiang Zhang, Hepatic Surgery Center, Tongji Hospital, Tongji Medical College, Huazhong University of Science and Technology, 1095 Jie Fang Avenue, Wuhan, Hubei 430030, P.R. China

E-mail: liangchu@tjh.tjmu.edu.cn

E-mail: bixiangzhang@163.com

Key words: liver kinase B1, hepatocellular carcinoma, prognostic biomarker ratio is high under energy stress conditions, and restores intracellular ATP levels by stimulating catabolic and inhibiting anabolic pathways (2). Studies on LKB1 in cancer have demonstrated its role as a master tumor suppressor in the majority of human cancer types, including melanoma (3), non-small-cell lung carcinoma (4) and other epithelial cancer types (5). However, recent studies have reported that LKB1 acts as a proto-oncogene in certain types of cancer. Bardeesy et al (6) indicated that $\mathrm{LKB1}^{-/-}$mouse embryonic fibroblasts were resistant to transformation by activated Ha-Ras, either alone or with immortalizing genes. Jeon et al (7) reported that knockdown of LKB1 and AMPK in breast cancer cells attenuated tumor development due to failure to inhibit acetyl-CoA carboxylase activity and to maintain intracellular NADPH levels. Furthermore, Martinez-Lopez et al (8) reported that glycine N-methyltransferase (GNMT) knockout mice may develop hepatocellular carcinoma (HCC). Reduced expression of GNMT in mouse and human HCC cells increased the activity of LKB1 and RAS. Lee et al (9) demonstrated that the stabilization and activation of LKB1/STE20-related kinase adaptor $\alpha$ (STRADA)/scaffolding mouse 25 (MO25) complex by S-phase kinase-associated protein 2-dependent ubiquitination was crucial for cell survival under energy stress conditions. They also indicated that LKB1 was highly expressed in late-stage $\mathrm{HCC}$ and its overexpression predicts poor survival outcomes. Furthermore, a study by Huang et al (10) suggested that the expression of LKB1 was decreased in HCC patients, and that low LKB1 expression predicted poor survival. Due to these contradicting results, the aim of the present study was to elucidate the role of LKB1 in $\mathrm{HCC}$.

\section{Materials and methods}

Patients, specimens and follow-up. In the present study, two independent cohorts of patients who underwent curative resection at the Hepatic Surgery Center of Tongji Hospital of Huazhong University of Science and Technology (Wuhan, China) between January 2004 and January 2014 were enrolled. For cohort 1, a total of 229 HCC tissues and matched surrounding analogous non-cancerous tissues (ANT) were collected for immunohistochemical (IHC) analysis; these patients were diagnosed with liver tumors, hepatectomy was 
performed and pathological analysis confirmed the diagnosis of HCC. Complete clinicopathological data and follow-up results were acquired for this cohort. Cohort 2, lacking follow-up data, included $60 \mathrm{HCC}$ samples and matched ANT for western blot analysis of LKB1 expression. Furthermore, the level of phosphorylated (p)-AMPK (Thr172) was measured to elucidate the activation of downstream signaling in 10 pairs of ANT and HCC samples. The preoperative diagnosis of HCC was performed according to the diagnostic criteria of the American Association for the Study of Liver Diseases (11). All the patients were followed-up until October 2014, with a median survival time of $23.30 \pm 0.97$ months. Overall survival was defined as the time interval between the date of surgery and the date of death or the last follow-up. Disease-free survival was defined as the time interval between the date of surgery and the date of recurrence confirmed by abdominal ultrasound examinations and serum $\alpha$-fetoprotein levels. If no recurrence was diagnosed, patients were censored on the date of death or the last follow-up. The median disease-free survival time was $17.02 \pm 0.98$ months. The present study was approved by the Ethics Committee of Tongji Hospital, Huazhong University of Science and Technology (Wuhan, China). The study protocol conformed to the principles outlined in the Declaration of Helsinki and written informed consent was obtained from each patient.

IHC. Formalin-fixed, paraffin-embedded tissues were sectioned at $2 \mu \mathrm{m}$, deparaffinized in xylene and rehydrated through a graded series of ethanol. Antigen retrieval was performed by microwave heating in $10 \mathrm{mM}$ Tris base and $1 \mathrm{mM}$ EDTA (pH 9.0). Endogenous peroxidase was blocked with $3 \% \mathrm{H}_{2} \mathrm{O}_{2}$ in methanol. The sections were then incubated with primary antibody at $4^{\circ} \mathrm{C}$ overnight (Table I). A Dako EnVision kit (Dako, Glostrup, Denmark) was used for incubation with the secondary antibody (Table I) and detection of peroxidase activity. Hematoxylin (Sigma-Aldrich; Merck KGaA, Darmstadt, Germany) was used to counterstain the nuclei for $5 \mathrm{~min}$ at room temperature. IHC scores were obtained by multiplying the percentage score with the intensity score of positively stained cells, as described previously (12). Scoring was performed by two certified pathologists independently, who were blinded to the patients' clinical and demographic information. The expression status is represented by the mean of several independent readings. An overall score of $>6$ and $\leq 6$ was considered to indicate high and low expression, respectively. The Edmondson-Steiner, Barcelona Clinic Liver Cancer (BCLC) and tumor-node-metastasis (TNM) stages were also determined $(13,14)$.

Cell lines and culture. The HCC cell lines MHCC97L, MHCC97H and HCCLM3 were obtained from the Liver Cancer Institute of Zhongshan Hospital (Fudan University, Shanghai, China). The HCC cell lines HLE and HLF were kindly provided by Shanshan Wang and Gang Li (Department of Molecular Biology, Peking University Health Science Center, Beijing, China). The hepatoblastoma cell line HepG2, and the HCC cell lines Hep3B, Huh7 and SK-Hep1 were purchased from the China Center for Type Culture Collection (Wuhan, China). The HCC cell line PLC/PRF-5 was purchased from the cell bank of the Chinese Academy of Sciences (Shanghai, China). All cell lines were maintained in Dulbecco's modified Eagle's medium (DMEM; Gibco; Thermo Fisher Scientific, Inc., Waltham, MA, USA) supplemented with $10 \%$ fetal bovine serum (Gibco; Thermo Fisher Scientific, Inc.) in a humidified atmosphere with $5 \% \mathrm{CO}_{2}$ at $37^{\circ} \mathrm{C}$.

Western blot analysis. HCC cell lines and samples were lysed in radioimmunoprecipitation assay buffer (Beyotime Institute of Biotechnology, Haimen, China) with proteinase and phosphatase inhibitor cocktail (Hoffmann-La Roche Ltd., Basel, Switzerland), and the protein concentration was determined by using Pierce ${ }^{\mathrm{TM}}$ BCA Protein Assay Kit (Thermo Fisher Scientific, Inc.). A total of $20 \mu \mathrm{g}$ of each protein was separated by $10 \%$ SDS-PAGE (Boster Biotechnology, Wuhan, China) and transferred to a polyvinylidene difluoride membrane (Hoffmann-La Roche Ltd.). The membrane was blocked with $5 \%$ skimmed milk dissolved by $1 \mathrm{X}$ Tris-buffered saline containing Tween-20 and incubated with specific primary antibodies at $4^{\circ} \mathrm{C}$ overnight (Table I), followed by incubation with a horseradish peroxidase (HRP)-conjugated anti-mouse or anti-rabbit secondary antibody (Jackson ImmunoResearch Laboratories, Inc., West Grove, PA, USA) at $37^{\circ} \mathrm{C}$ for $2 \mathrm{~h}$ (Table I). Detection was performed using a ChemiDoc ${ }^{\mathrm{TM}}$ Imaging System (Bio-Rad Laboratories Inc., Hercules, CA, USA).

Lentivirus production, transfection and establishment of stable cell clones. The pLKO.1-TRC cloning vector (cat. no. 10878) was from Addgene, Inc. (Cambridge, MA, USA). Small hairpin (sh)RNA specific for LKB1 (shLKB1) oligos were synthesized by Tsingke Technology (Wuhan, China) and were inserted into the pLKO.1 vector, which was then transfected into 293 cells with psPAX2 and pMD2.G (cat. nos. 12260 and 12259, respectively; Addgene, Inc.) using X-tremeGENE ${ }^{\mathrm{TM}}$ HP DNA Transfection Reagent (Sigma-Aldrich; Merck KGaA). After $48 \mathrm{~h}$ of incubation, the virus-containing supernatant was collected and filtered through a $0.45-\mu \mathrm{m}$ filter (PALL, Port Washington, NY, USA) (15). LKB1 overexpression lentivirus was purchased from Genecreate Technology (Wuhan, China). HCC cells were transfected with lentiviral particles in the presence of $8 \mu \mathrm{g} / \mathrm{ml}$ polybrene (Sigma-Aldrich; Merck $\mathrm{KGaA}$ ) with a multiplicity of infection (MOI) ranging from 50 to 100 . At $72 \mathrm{~h}$ after transfection, cells were selected with $5 \mu \mathrm{g} / \mathrm{ml}$ puromycin (Merck Calbiochem, Darmstadt, Germany) for 2 weeks. Selected pools of LKB1-knockdown or overexpressing cells were used for the subsequent experiments. The shRNA sequences are listed in Table II. HCC-LM3 shLKB1 and Huh7 shLKB1 refer to the HCC cell lines transfected with shLKB1 (LKB1 knockdown), whereas HLF LKB1 refers to the cell lines transfected with LKB1 overexpression virus. Control cells were transfected with empty vector.

Cell proliferation assay. HCC-LM3 shLKB1, HLF-LKB1 cells $\left(1 \times 10^{3}\right.$ cells/well) or Huh-7 shLKB1 cells $\left(3 \times 10^{3}\right.$ cells/well $)$ and the same amount of control cells were seeded into 96-well plates. At the indicated time points, Cell Counting Kit- 8 reagent (Beyotime Institute of Biotechnology) was added, followed by incubation for $1 \mathrm{~h}$ at $37^{\circ} \mathrm{C}$. The plate was read 
Table I. Antibodies used in this study.

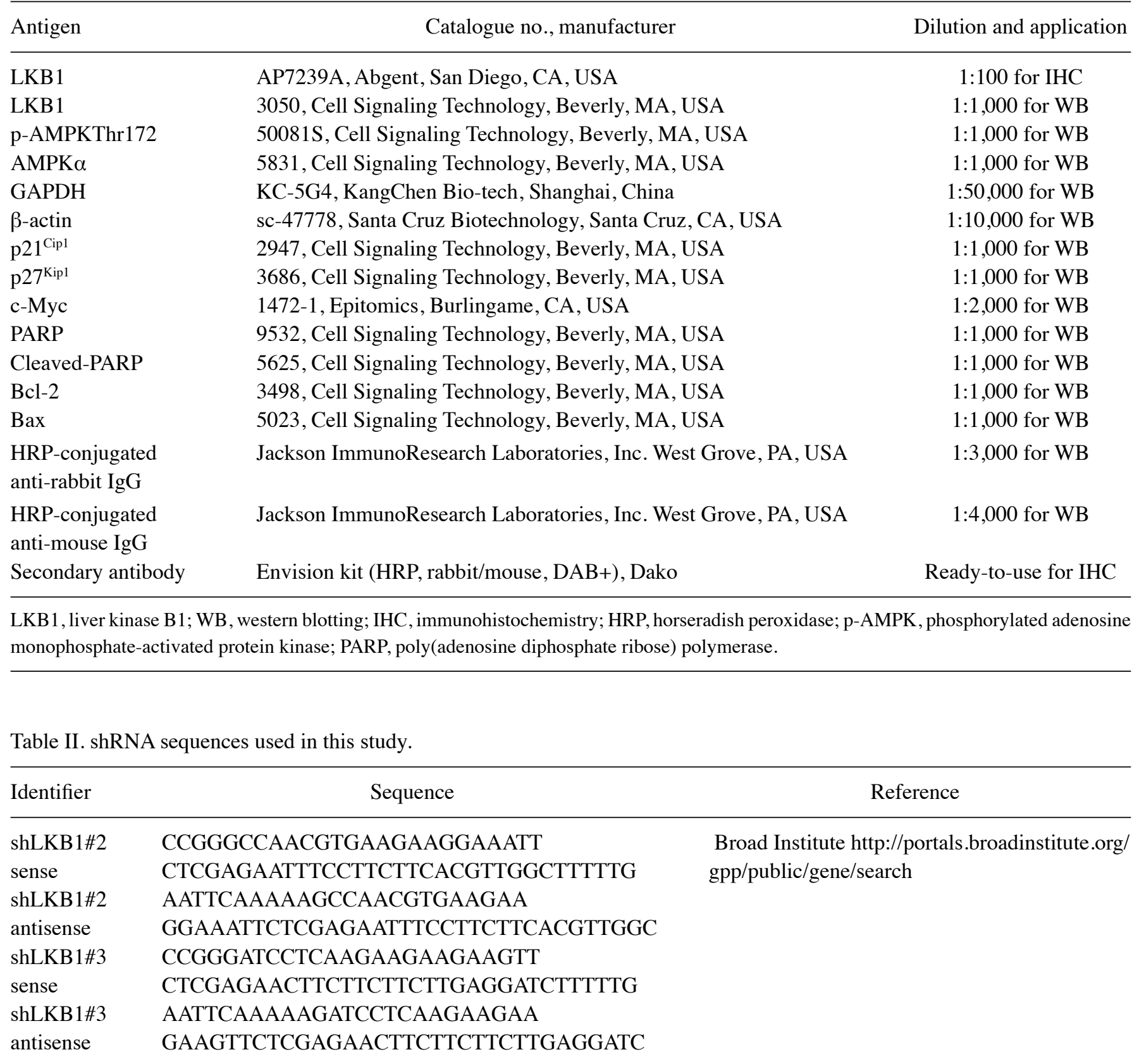

using an ELISA plate reader (Elx 800; Bio-Tek, Winooski, VT, USA) at a wavelength of $450 \mathrm{~nm}$. Experiments were repeated three times.

Colony formation assay. Transfected or control cells were seeded into 6-well plates at 500 cells/well, and the medium was changed every 3 days. After 10 days of incubation, the cells were fixed with $4 \%$ formalin and stained with $0.1 \%$ crystal violet solution (ServiceBio Technology, Wuhan, China). The numbers of colonies $>100 \mu \mathrm{m}$ in diameter were quantified with a ChemiDoc ${ }^{\mathrm{TM}}$ Imaging System (Bio-Rad Laboratories, Inc.). The experiments were repeated three times.

Apoptosis assay. Huh-7 shLKB1, HCC-LM3 shLKB1 or the corresponding control cells were seeded into 6-well plates at
$5 \times 10^{5}$ cells/well. After the cells were attached to the culture dish and had entered the logarithmic proliferation phase, they were thoroughly trypsinized, suspended, washed with icecold phosphate-buffered saline 3 times, re-suspended with $1 \mathrm{X}$ binding buffer, incubated with Annexin V and 7-aminoactinomycin D (BD Biosciences, Franklin Lakes, NJ, USA) at $37^{\circ} \mathrm{C}$ for $15 \mathrm{~min}$, and analyzed with a BD FACSCalibur (BD Biosciences). Experiments were repeated three times.

In vivo tumorigenicity assay. HCC-LM3 shLKB1 cells $\left(2 \times 10^{6}\right)$, Huh-7 shLKB1 cells $\left(5 \times 10^{6}\right)$, HLF-LKB1 cells $\left(1 \times 10^{6}\right)$ and equal amounts of the corresponding control cells were suspended in $100 \mu \mathrm{l}$ DMEM and subcutaneously injected into the flank of 5-week-old male nude mice (weight, 18-19 g). All the experimental mice were purchased from HFK Technology 
A
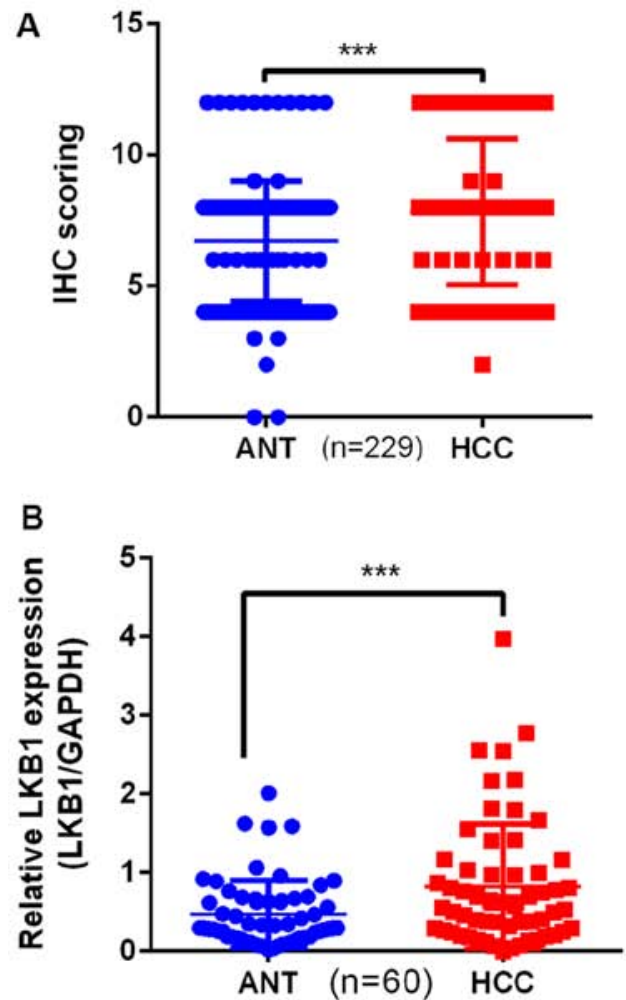
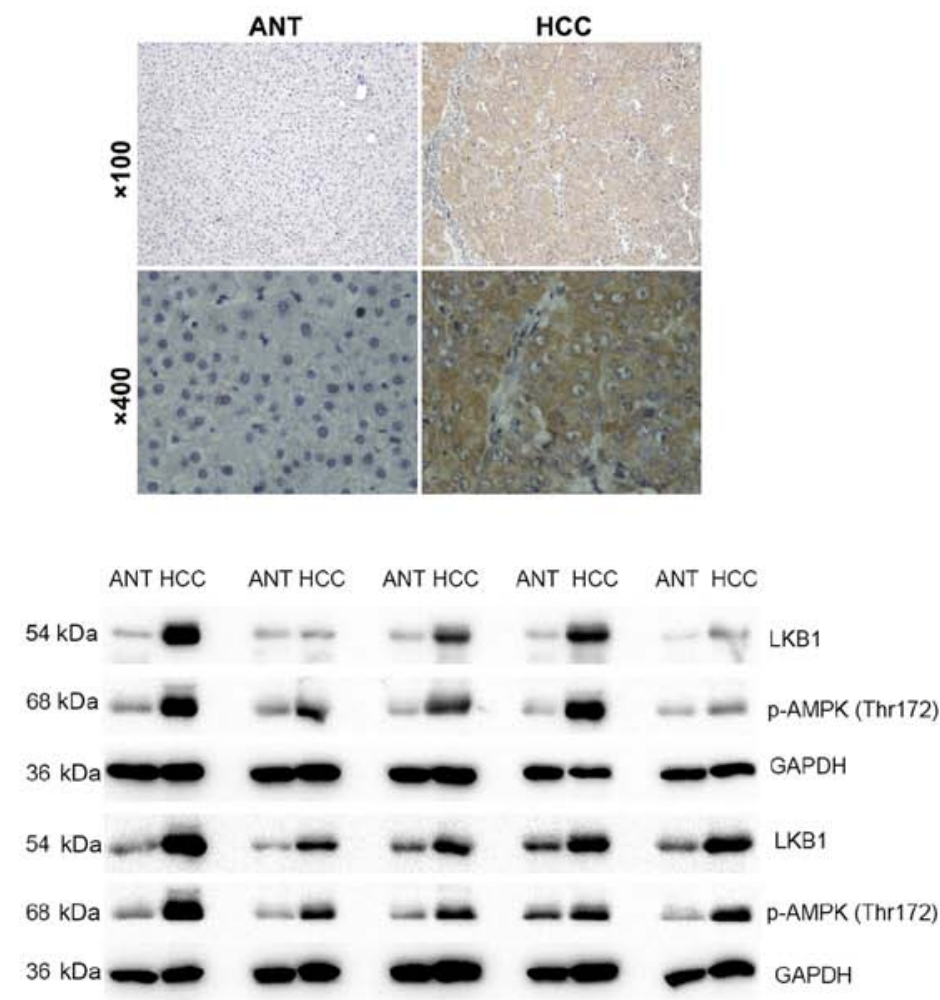

Figure 1. LKB1 is upregulated in HCC. (A) Immunohistochemical staining and (B) western blot analysis of LKB1 expression and p-AMPK (Thr172) levels in paired HCC and ANT tissues. Representative images (right panel) and quantified results (right panel) are provided. Values are expressed as the mean \pm standard deviation ( $\mathrm{n}=229$ in $\mathrm{A}, \mathrm{n}=60$ in $\mathrm{B}$ ). ${ }^{* * *} \mathrm{P}<0.001$. HCC, hepatocellular carcinoma; LKB1, liver kinase B1; p-AMPK, phosphorylated adenosine monophosphateactivated protein kinase; ANT, analogous non-cancerous tissues.

(Beijing, China) and kept under specific pathogen-free conditions with free access to food and water. The experimental mice were routinely monitored and sacrificed at the indicated time points. The length and width of the tumors was manually monitored using a Vernier caliper. Tumor volume (V) was calculated according to the following equation: $\mathrm{V}\left(\mathrm{mm}^{3}\right)$ $=0.5 \times \mathrm{L} \mathrm{x} \mathrm{W}^{2}$, where $\mathrm{L}$ is the length and $\mathrm{W}$ the width in $\mathrm{mm}$ (16). The animal experiments were approved by the Ethics Committee of Tongji Hospital, Huazhong University of Science and Technology (Wuhan, China).

Statistical analysis. Statistical analysis was performed using SPSS 19.0 (IBM Corp., Armonk, NY, USA) or Prism 6.0 (GraphPad Software, Inc., La Jolla, CA, USA) software. Comparison between groups was performed by a two-tailed Student's t-test, analysis of variance with Bonferroni's post hoc test, Chi-squared test, Spearman's correlation coefficient test or a non-parametric test, including the Wilcoxon's signed-rank test. Kaplan-Meier analysis and the log-rank test were used to compare the survival between subgroups. A Cox proportional hazards model was used for univariate and multivariate analyses to determine the factors independently associated with survival and recurrence. $\mathrm{P}<0.05$ was considered to indicate a statistically significant difference.

\section{Results}

LKB1 expression is upregulated in HCC. To determine the clinical significance of LKB1 in the development of HCC, the expression pattern of LKB1 was examined in two cohorts of patients. Cohort 1 included 229 patients (Table III) and cohort 2 included 60 patients. The expression of LKB1 was examined by IHC in matched pairs of HCC and ANT specimens in cohort 1. The results indicated that the expression levels of LKB1 were significantly higher in HCC tissues $(8.288 \pm 2.922)$ compared with those in ANT tissues $(6.716 \pm 2.293$; Fig. 1A). This was further confirmed by western blot analysis in specimens from cohort 2: The intensity ratio (LKB1/GAPDH) in HCC tissues (0.8236 \pm 0.7931$)$ was significantly higher compared with that in ANT tissues (0.4727士0.4279; Fig. 1B). p-AMPK (Thr172) was also upregulated in samples with high expression of LKB1 (Fig. 1B). These results suggested that LKB1 may play a protooncogenic role in $\mathrm{HCC}$.

Upregulated LKB1 expression is correlated with numerous malignant characteristics and poor prognosis. Due to the evidence supporting the possible proto-oncogenic role of LKB1 in HCC, the present study then aimed to further elucidate the correlation between LKB1 expression and clinical characteristics. Upregulation of LKB1 was significantly $(\mathrm{P}<0.001)$ correlated with several clinicopathological characteristics associated with aggressive biological behavior of cancer cells, including higher number of tumor foci, larger tumor size, incomplete tumor encapsulation, vascular invasion, portal vein tumor thrombus (PVTT), poor differentiation, advanced Edmondson-Steiner grade, advanced BCLC grade and TNM stage (Fig. 2A-H). Most importantly, upregulated LKB1 expression was correlated with a shorter overall 
Table III. Correlation between LKB1 expression and clinicopathological characteristics in 229 HCC patients.

\begin{tabular}{|c|c|c|c|c|}
\hline Clinicopathological variables & Low expression & High expression & Percentage $(\%)$ & P-value \\
\hline \multicolumn{5}{|l|}{ Sex } \\
\hline Male & 45 & 161 & 89.96 & \multirow[t]{2}{*}{$0.643^{\mathrm{a}}$} \\
\hline Female & 6 & 17 & 10.04 & \\
\hline \multicolumn{5}{|l|}{ Age, years } \\
\hline$\leq 50$ & 28 & 86 & 49.78 & \multirow[t]{2}{*}{$0.297^{b}$} \\
\hline$>50$ & 23 & 92 & 50.22 & \\
\hline \multicolumn{5}{|l|}{$\mathrm{BMI}, \mathrm{kg} / \mathrm{m}^{2}$} \\
\hline$<25$ & 37 & 124 & 70.31 & \multirow[t]{3}{*}{$0.763^{b}$} \\
\hline$\geq 25$ & 14 & 53 & 29.26 & \\
\hline Missing & & 1 & 0.44 & \\
\hline \multicolumn{5}{|l|}{ Alcohol intake } \\
\hline Yes & 14 & 61 & 32.75 & \multirow[t]{3}{*}{0.469} \\
\hline No & 37 & 115 & 66.38 & \\
\hline Missing & & 2 & 0.87 & \\
\hline \multicolumn{5}{|l|}{ Smoking } \\
\hline Current, past & 12 & 81 & 40.61 & \multirow[t]{3}{*}{0.015} \\
\hline Never & 39 & 96 & 58.95 & \\
\hline Missing & & 1 & 0.44 & \\
\hline \multicolumn{5}{|l|}{ HBV } \\
\hline Negative & 3 & 16 & 8.30 & \multirow[t]{3}{*}{0.466} \\
\hline Positive & 48 & 160 & 90.83 & \\
\hline Missing & & 2 & 0.87 & \\
\hline \multicolumn{5}{|l|}{ Cirrhosis } \\
\hline Absent & 10 & 39 & 21.40 & \multirow[t]{2}{*}{0.724} \\
\hline Present & 41 & 139 & 78.60 & \\
\hline \multicolumn{5}{|l|}{ Tumor number } \\
\hline Single & 44 & 137 & 79.04 & \multirow[t]{2}{*}{0.150} \\
\hline Multiple & 7 & 41 & 20.96 & \\
\hline \multicolumn{5}{|l|}{ Tumor size, $\mathrm{cm}$} \\
\hline$\leq 5$ & 25 & 64 & 38.86 & \multirow[t]{3}{*}{$0.005^{b}$} \\
\hline$>5$ & 26 & 112 & 60.26 & \\
\hline Missing & & 2 & 0.87 & \\
\hline Tumor encapsulation & & & & \\
\hline None & 14 & 91 & 45.85 & 0.009 \\
\hline Complete & 37 & 86 & 53.71 & \\
\hline Missing & & 1 & 0.44 & \\
\hline Vascular invasion & & & & \\
\hline Unidentified & 44 & 113 & 68.56 & 0.002 \\
\hline Identified & 7 & 65 & 31.44 & \\
\hline PVTT & & & & \\
\hline Unidentified & 46 & 132 & 77.73 & 0.015 \\
\hline Identified & 5 & 46 & 22.27 & \\
\hline Local invasion & & & & \\
\hline Unidentified & 49 & 166 & 93.88 & $0.738^{\mathrm{a}}$ \\
\hline Identified & 2 & 11 & 5.68 & \\
\hline Missing & & 1 & 0.44 & \\
\hline Distant metastasis & & & & \\
\hline Absent & 51 & 168 & 95.63 & $0.214^{\mathrm{a}}$ \\
\hline Present & 0 & 9 & 3.93 & \\
\hline Missing & & 1 & 0.44 & \\
\hline Differentiation & & & & \\
\hline Poor & 17 & 64 & 35.371 & $\mathbf{0 . 0 2} 2^{\mathrm{b}}$ \\
\hline Moderate & 23 & 86 & 47.598 & \\
\hline High & 11 & 28 & 17.031 & \\
\hline Edmondson-Steiner grade & & & & \\
\hline I-II & 22 & 63 & 37.12 & 0.313 \\
\hline III-IV & 29 & 115 & 62.88 & \\
\hline
\end{tabular}


Table III. Continued.

\begin{tabular}{|c|c|c|c|c|}
\hline Clinicopathological variables & Low expression & High expression & Percentage $(\%)$ & P-value \\
\hline \multicolumn{5}{|l|}{ Child-Pugh stage } \\
\hline A & 47 & 135 & 79.47 & \multirow[t]{3}{*}{$0.016{ }^{a}$} \\
\hline $\mathrm{B}$ & 4 & 42 & 20.09 & \\
\hline Missing & & 1 & 0.44 & \\
\hline \multicolumn{5}{|l|}{ TNM stage } \\
\hline I-II & 46 & 134 & 78.60 & \multirow[t]{2}{*}{$0.021^{\mathrm{a}}$} \\
\hline III-IV & 5 & 44 & 21.40 & \\
\hline \multicolumn{5}{|l|}{ BCLC stage } \\
\hline $0-\mathrm{A}$ & 37 & 80 & 51.09 & \multirow[t]{3}{*}{$<0.001^{b}$} \\
\hline $\mathrm{B}$ & 4 & 17 & & \\
\hline $\mathrm{C}$ & 10 & 81 & & \\
\hline \multicolumn{5}{|l|}{ Fasting glucose level, $\mathrm{mM}$} \\
\hline$\leq 6.1$ & 34 & 132 & 72.49 & \multirow[t]{3}{*}{$0.934^{\mathrm{b}}$} \\
\hline$>6.1$ & 12 & 25 & 16.16 & \\
\hline Missing & 5 & 21 & 11.35 & \\
\hline \multicolumn{5}{|l|}{ Diabetes } \\
\hline Yes & 15 & 23 & 16.59 & \multirow[t]{2}{*}{0.005} \\
\hline No & 36 & 155 & 83.41 & \\
\hline \multicolumn{5}{|l|}{ ALT, U/1 } \\
\hline$\leq 40$ & 31 & 110 & 61.57 & \multirow[t]{3}{*}{$0.845^{b}$} \\
\hline$>40$ & 19 & 63 & 35.81 & \\
\hline Missing & 1 & 5 & 2.62 & \\
\hline \multicolumn{5}{|l|}{$\mathrm{AST}, \mathrm{U} / \mathrm{l}$} \\
\hline$\leq 40$ & 32 & 104 & 59.39 & \multirow[t]{3}{*}{$0.264^{\mathrm{b}}$} \\
\hline$>40$ & 17 & 66 & 36.24 & \\
\hline Missing & 2 & 8 & 4.37 & \\
\hline \multicolumn{5}{|l|}{ TBIL, $\mu \mathrm{M}$} \\
\hline$\leq 17.1$ & 41 & 128 & 73.80 & \multirow{3}{*}{$0.181^{\mathrm{b}}$} \\
\hline$>17.1$ & 9 & 43 & 22.71 & \\
\hline Missing & 1 & 7 & 3.49 & \\
\hline \multicolumn{5}{|l|}{$\gamma$-GGT, U/1 } \\
\hline$\leq 50$ & 19 & 55 & 32.31 & \multirow{3}{*}{$0.007^{b}$} \\
\hline$>50$ & 28 & 109 & 59.83 & \\
\hline Missing & 4 & 14 & 7.86 & \\
\hline \multicolumn{5}{|l|}{$\mathrm{AFP}, \mu \mathrm{g} / \mathrm{l}$} \\
\hline$\leq 20$ & 18 & 53 & 31.0 & \multirow[t]{3}{*}{$0.442^{b}$} \\
\hline$>20$ & 29 & 117 & 63.76 & \\
\hline Missing & 4 & 8 & 5.24 & \\
\hline CEA (ng/ml) & & & & \\
\hline$\leq 5.9$ & 46 & 138 & 80.35 & $0.744^{\mathrm{b}}$ \\
\hline$>5.9$ & 1 & 10 & 4.80 & \\
\hline Missing & 4 & 30 & 14.85 & \\
\hline CA19-9, U/ml & & & & \\
\hline$\leq 40$ & 41 & 138 & 78.17 & $0.501^{\mathrm{b}}$ \\
\hline$>40$ & 5 & 12 & 7.42 & \\
\hline Missing & 5 & 28 & 14.41 & \\
\hline Adjuvant TACE & & & & \\
\hline Yes & 4 & 12 & 6.99 & $0.760^{\mathrm{a}}$ \\
\hline No & 47 & 166 & 93.01 & \\
\hline Entecavir therapy & & & & \\
\hline Yes & 26 & 98 & 54.15 & 0.607 \\
\hline No & 25 & 80 & 45.85 & \\
\hline
\end{tabular}

aaisher's exact test. 'Spearman's correlation coefficient test. Other variables, Chi-squared test. Bold print indicates statistical significance. BMI, body mass index;TNM,tumor-node-metastasis; BCLC, Barcelona Clinic LiverCancer; PVTT, portal vein tumor thrombus; HBV, hepatitis B virus; AST, glutamic oxalacetic transaminase; ALT, glutamic-pyruvic transaminase; TBIL, total bilirubin; $\gamma$-GGT, $\gamma$-glutamyltranspeptidase; AFP, $\alpha$-fetoprotein; CEA, carcinoembryonic antigen; CA199, carbohydrate antigen 199; TACE, transcatheter arterial chemoembolization; LKB1, liver kinase B1. 
A

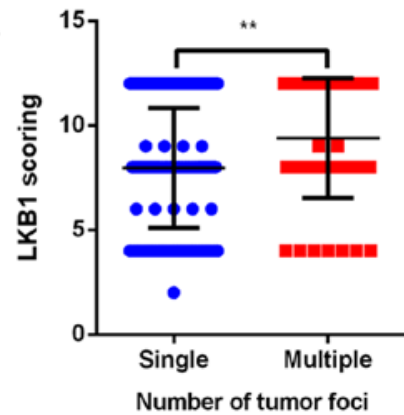

D
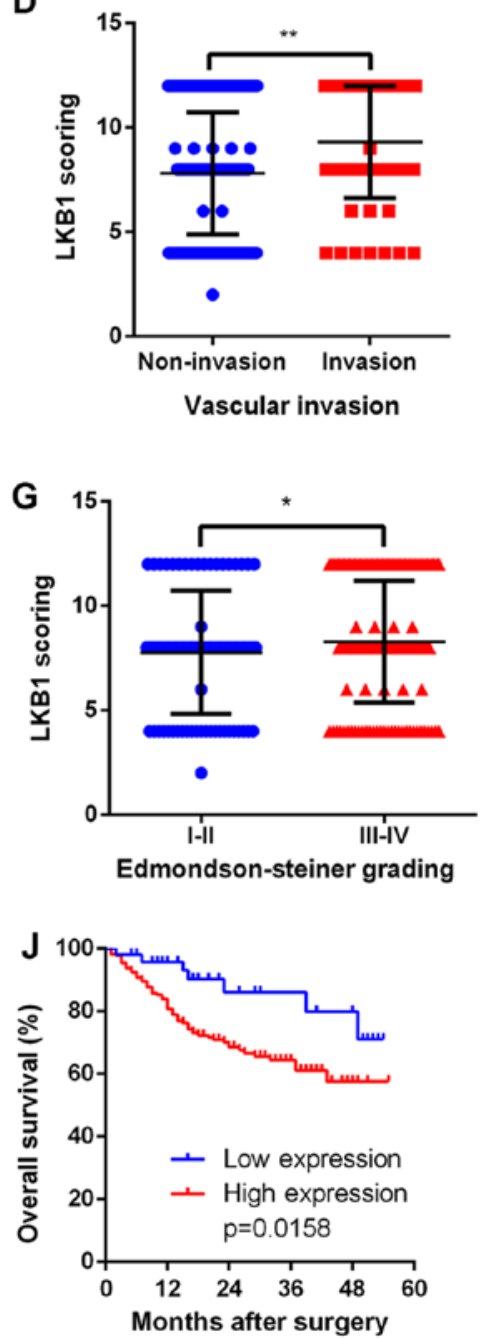

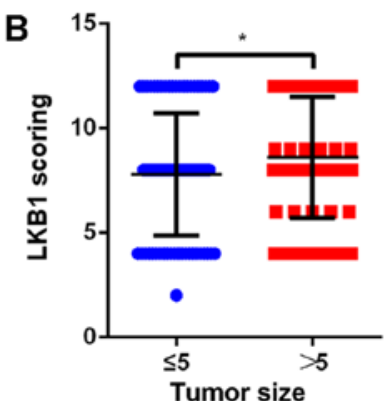

E
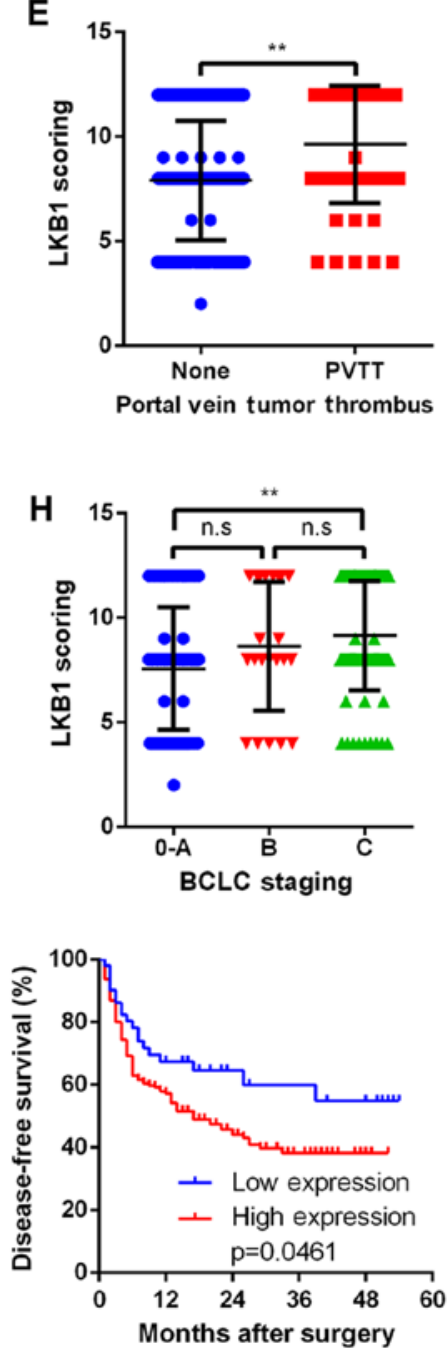
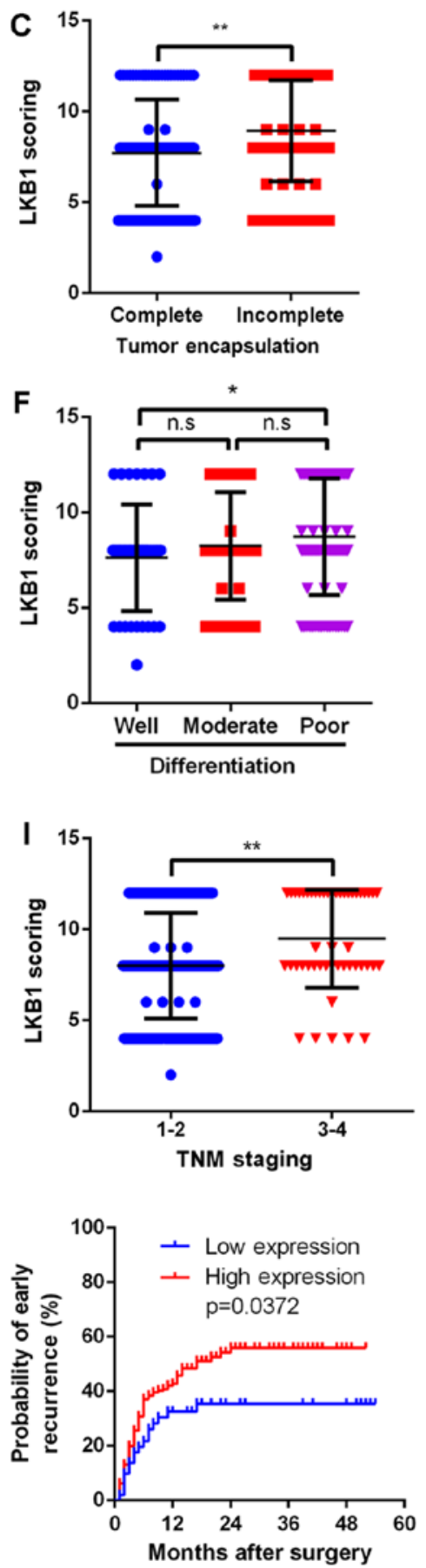

Figure 2. Upregulated LKB1 expression predicts aggressive clinicopathological characteristics and poor prognosis in HCC patients. Relative expression scores of LKB1 in 229 human HCC samples divided by (A) number of tumor foci, (B) tumor size, (C) tumor encapsulation, (D) vascular invasion, (E) PVTT, (F) tumor differentiation, $(\mathrm{G})$ Edmondson-Steiner grade, $(\mathrm{H})$ BCLC stage and (I) TNM stage. All values are presented as dot plots, with the middle bars representing the median and vertical bars representing the range of data. (J) Kaplan-Meier analysis of the correlation between LKB1 expression and the OS and DFS of HCC patients as well as early recurrence. ${ }^{* *} \mathrm{P}<0.01,{ }^{*} \mathrm{P}<0.05$; n.s, non-significant. HCC, hepatocellular carcinoma; LKB1, liver kinase B1; PVTT, portal vein tumor thrombus; BCLC, Barcelona Clinic Liver Cancer; TNM, tumor-node-metastasis.

survival $(\mathrm{P}=0.0158)$, shorter disease-free survival $(\mathrm{P}=0.0461)$ and higher early recurrence $(\mathrm{P}=0.0372$; Fig. $2 \mathrm{~J})$.

Furthermore, a large tumor size, multiple tumor foci, incomplete tumor encapsulation, PVTT, local invasion, vascular invasion, advanced BCLC or TNM stage were correlated with poorer survival (Fig. 3). Univariate and multivariate analyses revealed that high LKB1 expression in HCC patients may serve as an independent prognostic marker for overall survival $(\mathrm{P}=0.018$ and 0.046 for uni- and multivariate analysis, respectively), whereas it had no significant predictive value regarding recurrence $(\mathrm{P}=0.054$ and 0.383 , respectively; Table IV).

Knockdown of LKB1 expression inhibits HCC cell proliferation. In order to examine the role of LKB1 in HCC cell lines, LKB1 expression was knocked down in Huh7 and HCC-LM3 cells (Fig. 4A), which exhibit high and moderate endogenous LKB1 expression, respectively (data not shown). The CCK-8 
Table IV. Univariate and multivariate analyses of prognostic factors in overall survival and recurrence.

A, Overall survival

\begin{tabular}{|c|c|c|c|c|c|c|}
\hline \multirow[b]{2}{*}{ Factors } & \multicolumn{3}{|c|}{ Univariate analysis } & \multicolumn{3}{|c|}{ Multivariate analysis } \\
\hline & HR & $95 \% \mathrm{CI}$ & P-value & HR & $95 \% \mathrm{CI}$ & P-value \\
\hline Age ( $>50$ vs. $\leq 50$ years) & 0.990 & $0.775-1.265$ & 0.938 & & & \\
\hline Sex (male vs. female) & 1.528 & $1.115-2.092$ & 0.008 & 1.155 & $0.290-4.604$ & 0.302 \\
\hline Diabetes mellitus (yes vs. no) & 1.57 & $0.677-3.641$ & 0.293 & & & \\
\hline Blood glucose (>6.1 vs. $\leq 6.1 \mathrm{mM})$ & 0.923 & $0.453-1.882$ & 0.826 & & & \\
\hline Tumor foci (multiple vs. single) & 0.850 & $0.641-1.128$ & 0.26 & & & \\
\hline Tumor size ( $>5$ vs. $\leq 5 \mathrm{~cm}$ ) & 3.270 & $1.779-6.011$ & $<0.001$ & 4.161 & $1.587-10.910$ & 0.004 \\
\hline Tumor encapsulation (none vs. complete) & 0.432 & $0.263-0.711$ & 0.001 & 1.403 & $0.708-2.779$ & 0.332 \\
\hline Differentiation (poor vs. high + moderate) & 2.224 & $1.246-3.970$ & 0.007 & 0.397 & $0.110-1.430$ & 0.158 \\
\hline Edmondson-Steiner grade (III+IV vs. I+II) & 0.832 & $0.639-1.804$ & 0.173 & & & \\
\hline TNM stage (III+IV vs. I+II) & 0.732 & $0.557-0.961$ & 0.025 & 0.591 & $0.227-1.538$ & 0.281 \\
\hline Child-Pugh stage (B vs. A) & 1.387 & $0.786-2.448$ & 0.259 & & & \\
\hline BCLC stage $(\mathrm{B}+\mathrm{C}$ vs. $0+\mathrm{A})$ & 0.596 & $0.460-0.772$ & $<0.001$ & 1.772 & $0.541-5.803$ & 0.344 \\
\hline PVTT (identified vs. unidentified) & 2.314 & $1.407-3.805$ & 0.001 & 2.291 & $0.778-6.750$ & 0.133 \\
\hline Vascular invasion (identified vs. unidentified) & 0.514 & $0.401-0.685$ & $<0.001$ & 1.155 & $0.290-4.604$ & 0.838 \\
\hline Local invasion (identified vs. unidentified) & 2.661 & $1.211-5.847$ & 0.015 & 3.126 & $0.880-11.102$ & 0.078 \\
\hline Distant metastasis (identified vs. unidentified) & 1.446 & $0.453-4.613$ & 0.533 & & & \\
\hline HBV (positive vs. negative) & 0.939 & $0.405-2.176$ & 0.883 & & & \\
\hline Cirrhosis (present vs. absent) & 0.795 & $0.567-1.114$ & 0.182 & & & \\
\hline AST (>40 vs. $\leq 40)$ & 2.168 & $1.135-3.574$ & 0.002 & 1.295 & $0.660-2.540$ & 0.452 \\
\hline$\gamma$-GGT $(>50$ vs. $\leq 50)$ & 1.994 & $1.090-3.648$ & 0.025 & 1.204 & $0.507-2.862$ & 0.674 \\
\hline $\operatorname{AFP}(\geq 20$ vs. $<20)$ & 2.196 & $1.170-4.124$ & 0.014 & 1.605 & $0.781-3.297$ & 0.198 \\
\hline CA199 (>40 vs. $\leq 40)$ & 2.752 & $1.344-5.634$ & 0.006 & 7.273 & $3.079-17.177$ & $<0.001$ \\
\hline LKB1 expression (high vs. low) & 2.617 & $1.179-5.808$ & 0.018 & 2.372 & $1.014-5.550$ & 0.046 \\
\hline
\end{tabular}

B, Recurrence

\begin{tabular}{|c|c|c|c|c|c|c|}
\hline \multirow[b]{2}{*}{ Factors } & \multicolumn{3}{|c|}{ Univariate analysis } & \multicolumn{3}{|c|}{ Multivariate analysis } \\
\hline & HR & $95 \% \mathrm{CI}$ & P-value & HR & $95 \%$ CI & P-value \\
\hline Age $(>50$ vs $\leq 50$ years $)$ & 0.855 & $0.595-1.23$ & 0.399 & & & \\
\hline Sex (male vs. female) & 0.912 & $0.502-1.657$ & 0.762 & & & \\
\hline Diabetes mellitus (yes vs. no) & 0.569 & $0.313-1.035$ & 0.065 & & & \\
\hline Blood glucose (>6.1 vs. $\leq 6.1 \mathrm{mM})$ & 0.828 & $0.492-1.392$ & 0.476 & & & \\
\hline Tumor foci (multiple vs. single) & 1.988 & $1.337-2.956$ & 0.001 & 1.295 & $0.610-2.749$ & 0.500 \\
\hline Tumor size ( $>5$ vs. $\leq 5 \mathrm{~cm}$ ) & 2.983 & $1.937-4.595$ & $<0.001$ & 2.599 & $1.539-4.391$ & $<0.001$ \\
\hline Tumor encapsulation (none vs. complete) & 2.220 & $1.533-3.215$ & $<0.001$ & 1.520 & $0.969-2.383$ & 0.068 \\
\hline Differentiation (poor vs. high + moderate) & 0.046 & $0.218-0.755$ & 0.004 & 1.212 & $0.867-1.692$ & 0.260 \\
\hline Edmondson-Steiner grade (III+IV vs. I+II) & 1.325 & $0.902-1.948$ & 0.152 & & & \\
\hline TNM stage (III+IV vs. I+II) & 2.510 & $1.694-3.719$ & $<0.001$ & 0.994 & $0.751-1.315$ & 0.964 \\
\hline Child-Pugh stage (B vs. A) & 1.146 & $0.736-1.785$ & 0.546 & & & \\
\hline BCLC stage $(\mathrm{B}+\mathrm{C}$ vs. $0+\mathrm{A})$ & 2.385 & $1.637-3.475$ & $<0.001$ & 0.825 & $0.587-1.160$ & 0.269 \\
\hline PVTT (identified vs. unidentified) & 0.369 & $0.249-0.547$ & $<0.001$ & 0.673 & $0.456-0.994$ & 0.047 \\
\hline Vascular invasion (identified vs. unidentified) & 2.045 & $1.407-2.971$ & $<0.001$ & 1.256 & $0.827-1.909$ & 0.285 \\
\hline Local invasion (identified vs. unidentified) & 3.619 & $1.975-6.632$ & $<0.001$ & 2.367 & $1.072-5.228$ & 0.033 \\
\hline Distant metastasis (identified vs. unidentified) & 1.593 & $0.699-3.632$ & 0.268 & & & \\
\hline HBV (positive vs. negative) & 0.848 & $0.466-1.540$ & 0.588 & & & \\
\hline Cirrhosis (identified vs. unidentified) & 1.234 & $0.776-1.963$ & 0.374 & & & \\
\hline $\operatorname{AST}(>40$ vs. $\leq 40)$ & 1.593 & $1.097-2.313$ & 0.014 & 1.363 & $0.862-2.155$ & 0.185 \\
\hline
\end{tabular}


Table IV. Continued.

\begin{tabular}{|c|c|c|c|c|c|c|}
\hline \multirow[b]{2}{*}{ Factors } & \multicolumn{3}{|c|}{ Univariate analysis } & \multicolumn{3}{|c|}{ Multivariate analysis } \\
\hline & HR & $95 \% \mathrm{CI}$ & P-value & HR & $95 \% \mathrm{CI}$ & $\mathrm{P}$-value \\
\hline$\gamma$-GGT (>50 vs. $\leq 50)$ & 1.861 & $1.203-2.800$ & 0.005 & 1.706 & $0.946-3.076$ & 0.076 \\
\hline $\operatorname{AFP}(\geq 20$ vs. $<20)$ & 1.352 & $0.897-2.038$ & 0.150 & & & \\
\hline CA199 (>40 vs. $\leq 40)$ & 1.644 & $0.877-3.082$ & 0.121 & & & \\
\hline LKB1 expression (high vs. low) & 1.622 & $0.992-2.652$ & 0.054 & 1.263 & $0.747-2.133$ & 0.383 \\
\hline
\end{tabular}

TNM, tumor-node-metastasis; BCLC, Barcelona Clinic Liver Cancer; PVTT, portal vein tumor thrombus; HBV, hepatitis B virus; AST, glutamic oxalacetic transaminase; $\gamma$-GGT, $\gamma$-glutamyltranspeptidase; AFP, $\alpha$-fetoprotein; CA199; carbohydrate antigen 199; LKB1, liver kinase B1
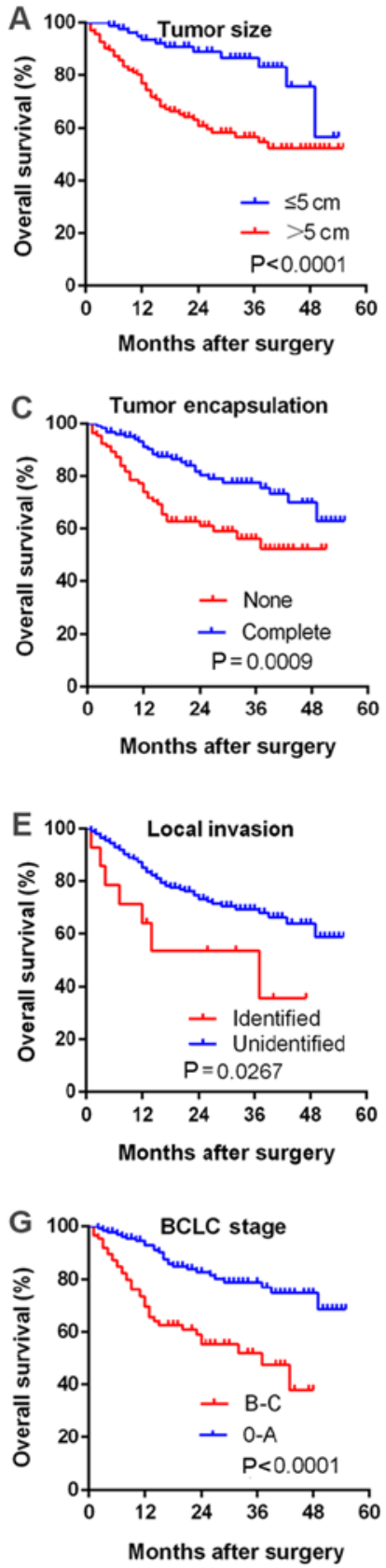
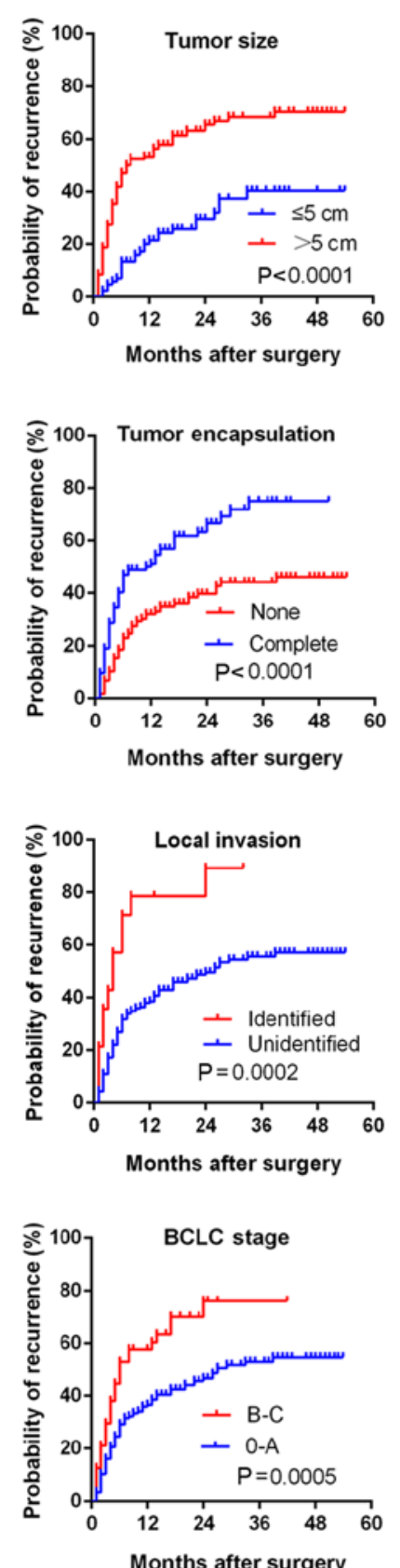
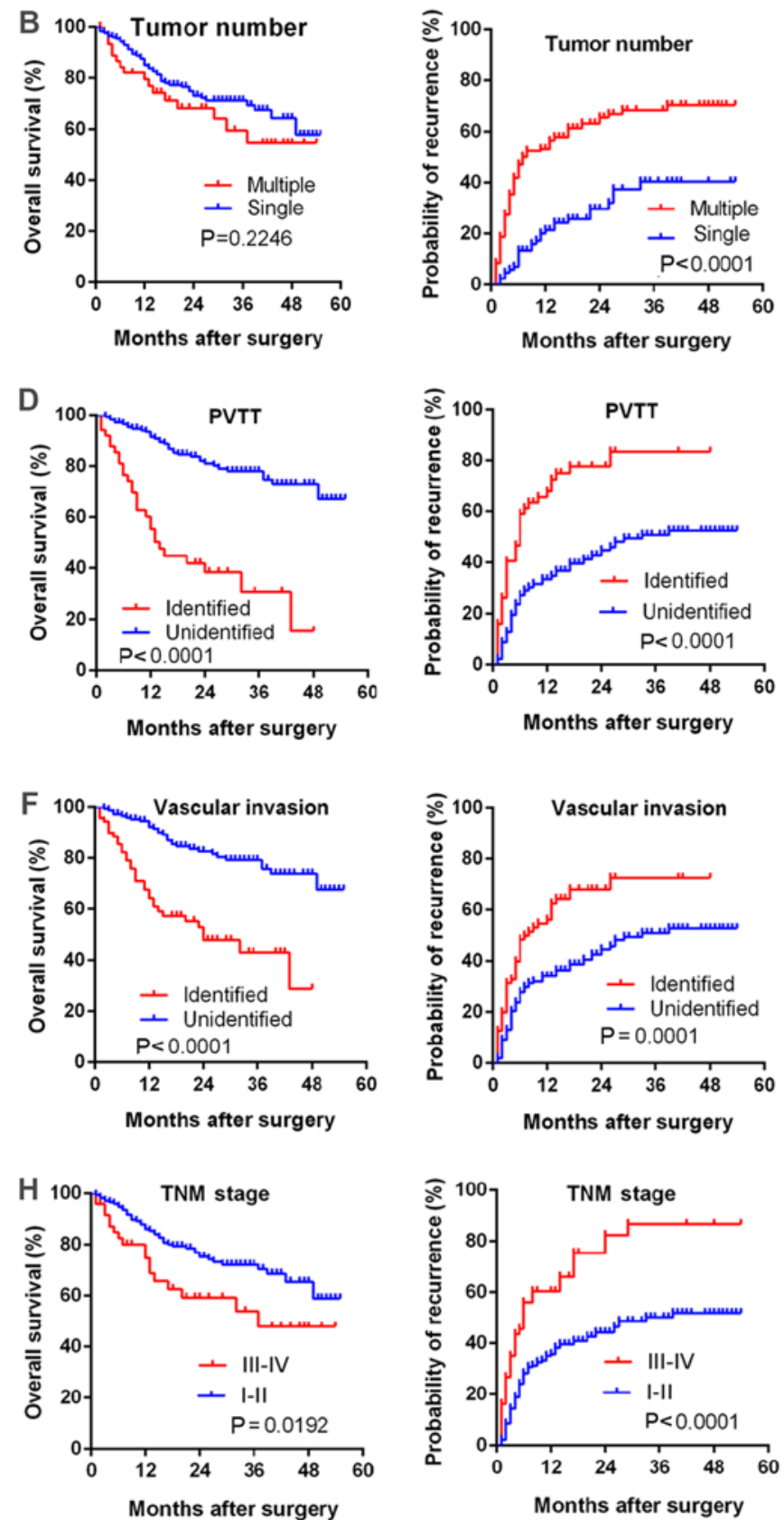

Figure 3. Aggressive pathological characteristics are correlated with poor prognosis. A total of 229 human hepatocellular carcinoma samples were stratified by (A) tumor size, (B) number of tumor foci, (C) state of tumor encapsulation, (D) presence of PVTT, (E) local invasion, (F) vascular invasion, (G) BCLC stage and $(\mathrm{H})$ TNM stage. All the pathological characteristics associated with aggressiveness predict shorter overall survival and earlier recurrence. PVTT, portal vein tumor thrombus; BCLC, Barcelona Clinic Liver Cancer; TNM, tumor-node-metastasis. 
A

Huh7

HCC-LM3

Mock Vec sh $\# 2$ sh\#3 Mock Vec sh $\# 2$ sh \#3

$54 \mathrm{kDa}$

L L

$36 \mathrm{kDa}$

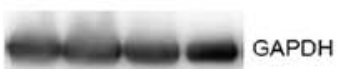

B

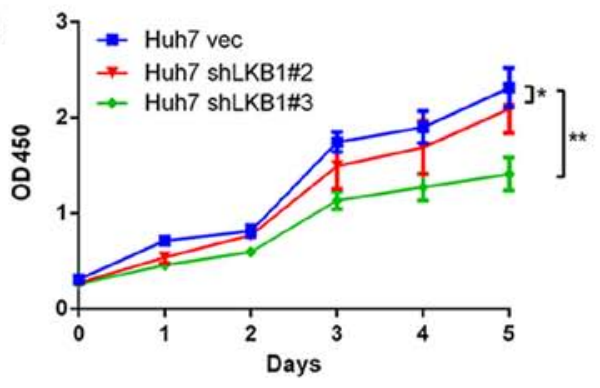

C
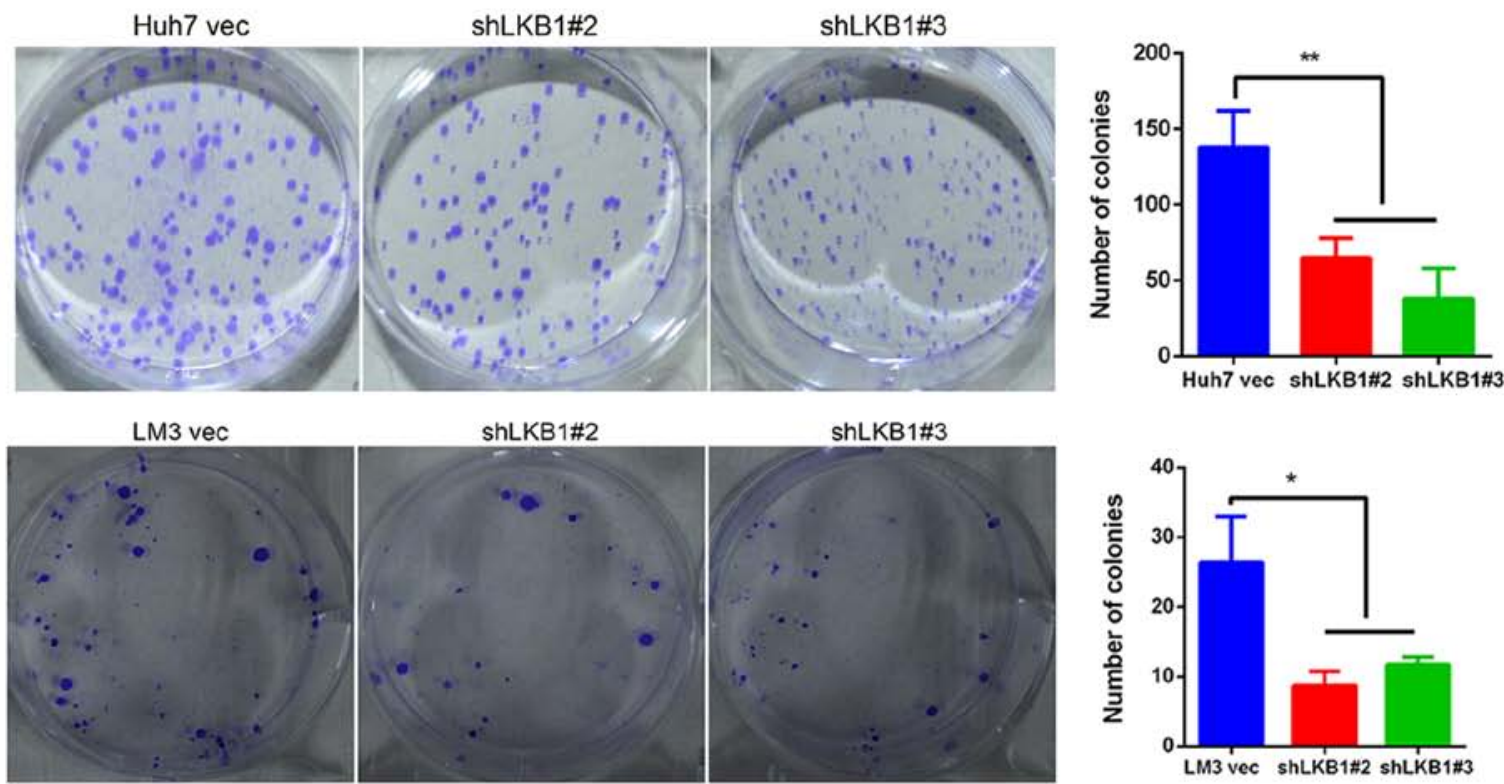

D
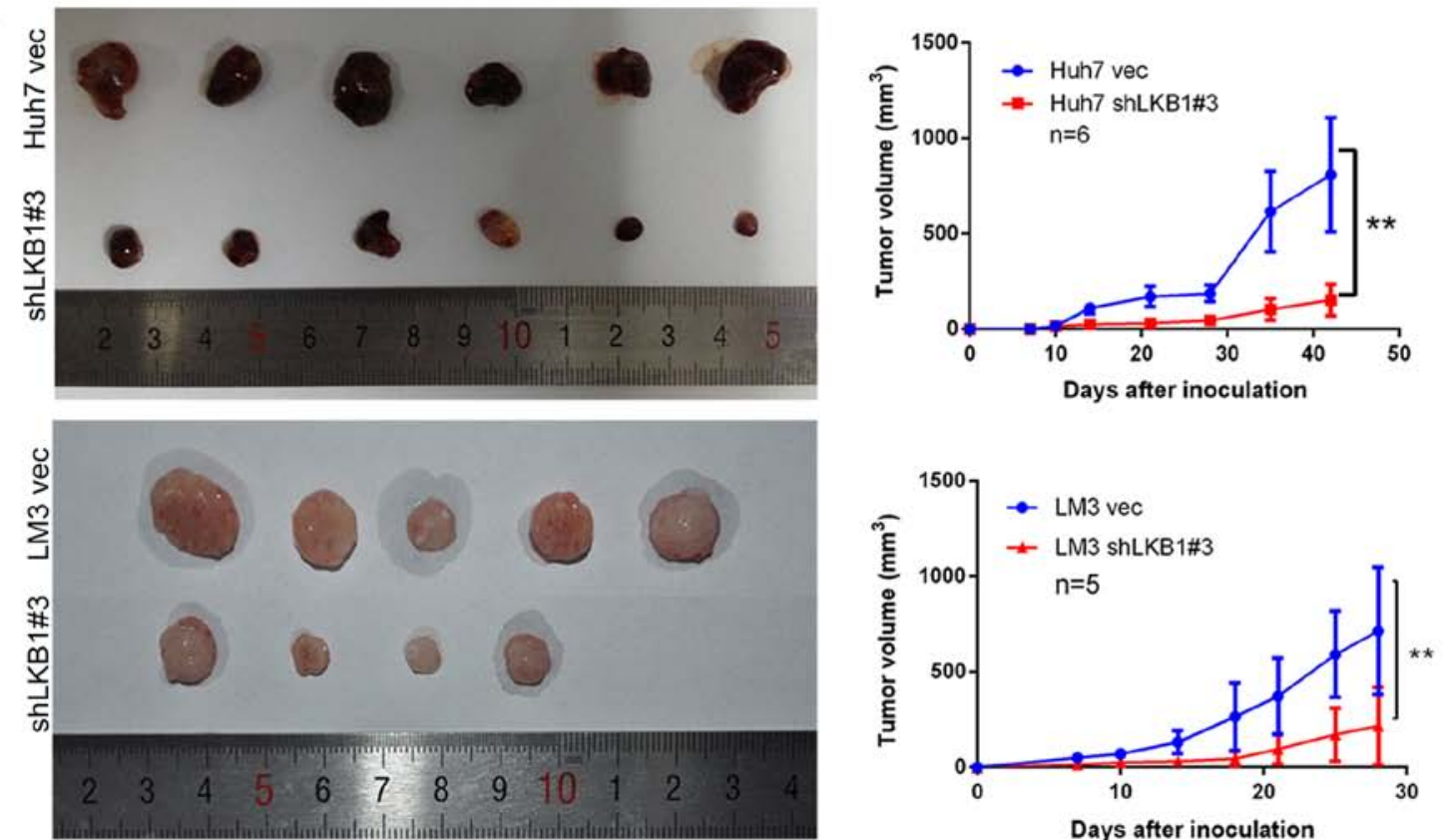

Figure 4. Knockdown of LKB1 expression suppresses the proliferation of HCC cells. (A) Western blot analysis confirmed knockdown of LKB1 expression and downregulation of p-AMPK (Thr172) by small hairpin RNAs in Huh7 and HCC-LM3 cells. (B and C) The effect of LKB1 on HCC cells was evaluated by (B) Cell Counting Kit-8 and (C) colony formation assay. Colony formation images representative of 3 independent experiments are provided. (D) Knockdown of LKB1 expression suppressed tumor growth by subcutaneously injected cells ( $\mathrm{n}=6$ and $\mathrm{n}=5$ in the Huh7 and HCC-LM3 groups, respectively). ${ }^{* *} \mathrm{P}<0.01$, ${ }^{*} \mathrm{P}<0.05$. HCC, hepatocellular carcinoma; LKB1, liver kinase B1; p-AMPK, phosphorylated adenosine monophosphate-activated protein kinase. 
A

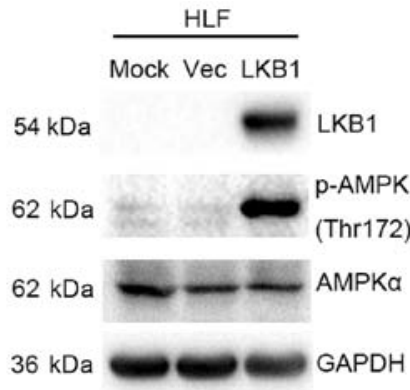

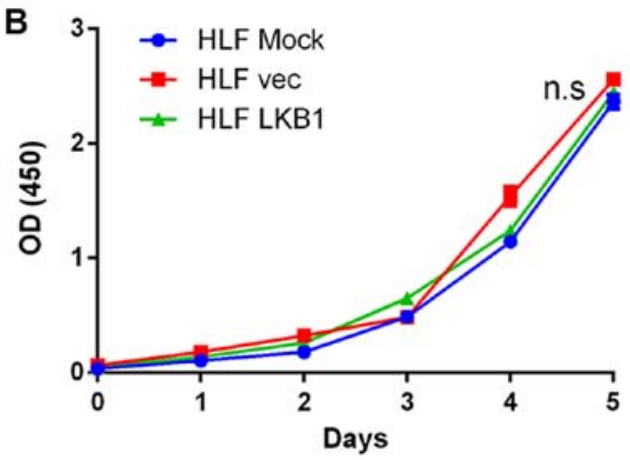

HLF LKB1

C
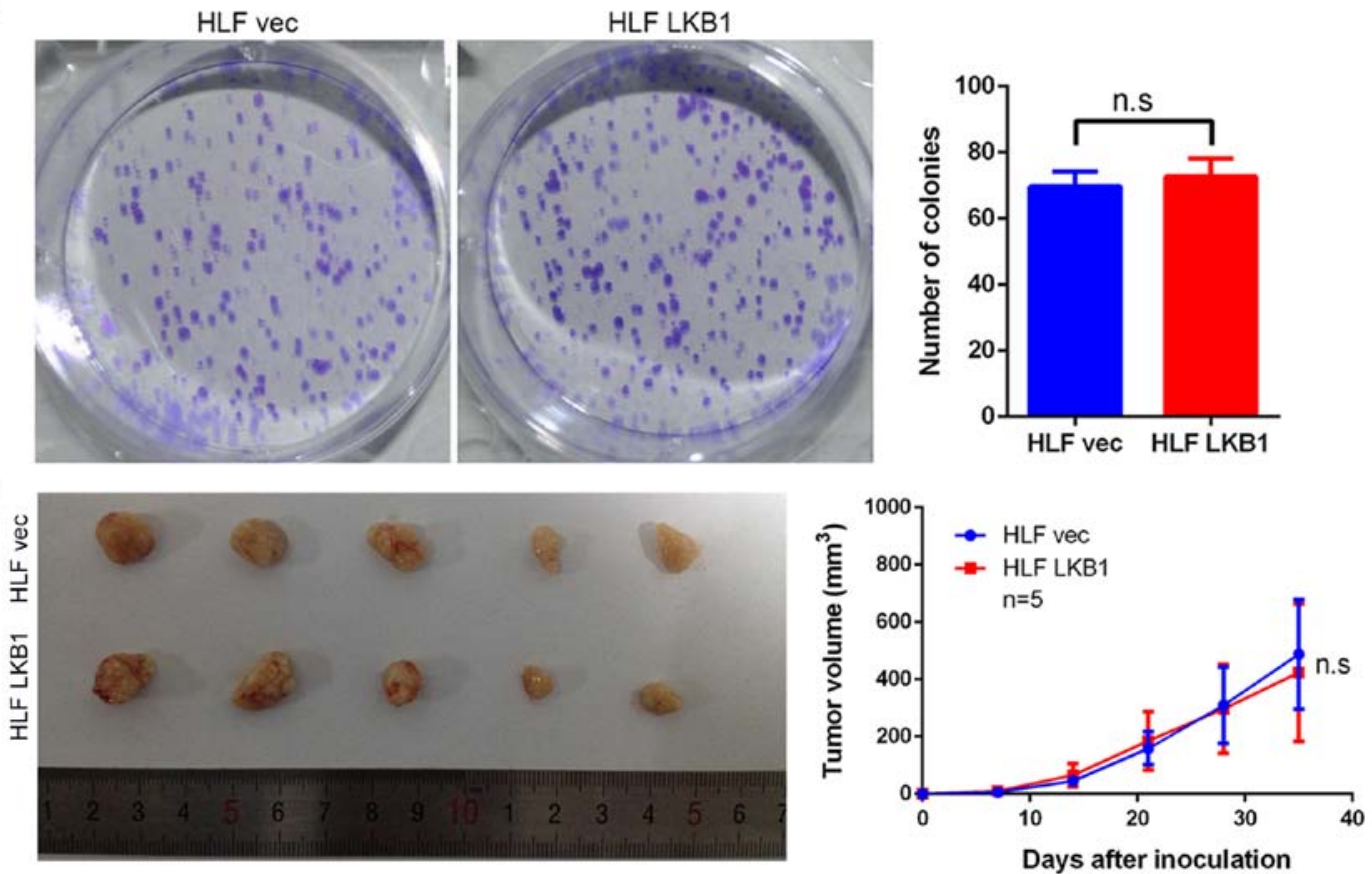

Figure 5. LKB1 overexpression did not affect the proliferation of HCC cells. (A) Western blot analysis confirmed the overexpression of LKB1 and upregulation of p-AMPK (Thr172) in HLF cells. (B and C) LKB1 overexpression exerted no effect on the proliferation of HCC cells as detected by (B) Cell Counting Kit-8 and (C) colony formation assay. Colony formation images representative of 3 independent experiments are provided. (D) LKB1 overexpression did not affect the growth of subcutaneously injected HLF cells ( $n=5)$. n.s, non-significant; HCC, hepatocellular carcinoma; LKB1, liver kinase B1; p-AMPK, phosphorylated adenosine monophosphate-activated protein kinase.

and colony formation assays indicated that knockdown of LKB1 significantly inhibited cell proliferation (Fig. 4B and C). Furthermore, LKB1 was found to be ectopically overexpressed in HLF cells (Fig. 5A), which have no detectable LKB1 expression (data not shown). However, overexpression of LKB1 exerted no effect on the growth of HLF cells (Fig. 5B and C). The in vivo tumorigenicity assay indicated that the volume of tumors grown from subcutaneously injected cells was smaller in the LKB1 knockdown groups compared with that in the control groups (Fig. 4D). However, no significant difference in volume was observed between the tumors derived from LKB1-overexpressing HLF and those from control cells (Fig. 5D).

LKB1 knockdown inhibits tumor cell proliferation by promoting cell apoptosis. Since knockdown of LKB1 inhibited Huh7 and HCC-LM3 cell proliferation, flow cytometric analysis was performed to determine whether this antiproliferative effect was due to cell cycle arrest. No significant differences in the distribution of cells in each phase of the cell cycle were observed (data not shown). However, the cell apoptosis assay indicated that, in LKB1-knockdown cells, the apoptotic rate was higher compared with that in the control cells (Fig. 6A). Western blot analysis further confirmed an increased amount of cleaved caspase- 3 and PARP in the knockdown group (Fig. 6B). In addition, reduced c-Myc expression and elevated expression of $\mathrm{p} 21^{\mathrm{Cip} 1}$ and $\mathrm{p} 27^{\mathrm{Kip} 1}$ were observed in LKB1-knockdown cells, suggesting that $\mathrm{p} 21^{\text {Cip1 }}$ and $\mathrm{p} 27^{\mathrm{Kip} 1}$ affect cell proliferation via other mechanisms (Fig. 6B).

\section{Discussion}

LKB1 has been reported to act as a tumor suppressor in the majority of published studies. LKB1 suppresses cell growth and viability through the LKB1/AMPK/mammalian target of rapamycin signaling pathway (17). However, certain studies suggested that LKB1 exerts a proto-oncogenic effect through modulating cellular metabolism and resistance to oncogenic transformation $(8,9)$. Therefore, it is of paramount importance 

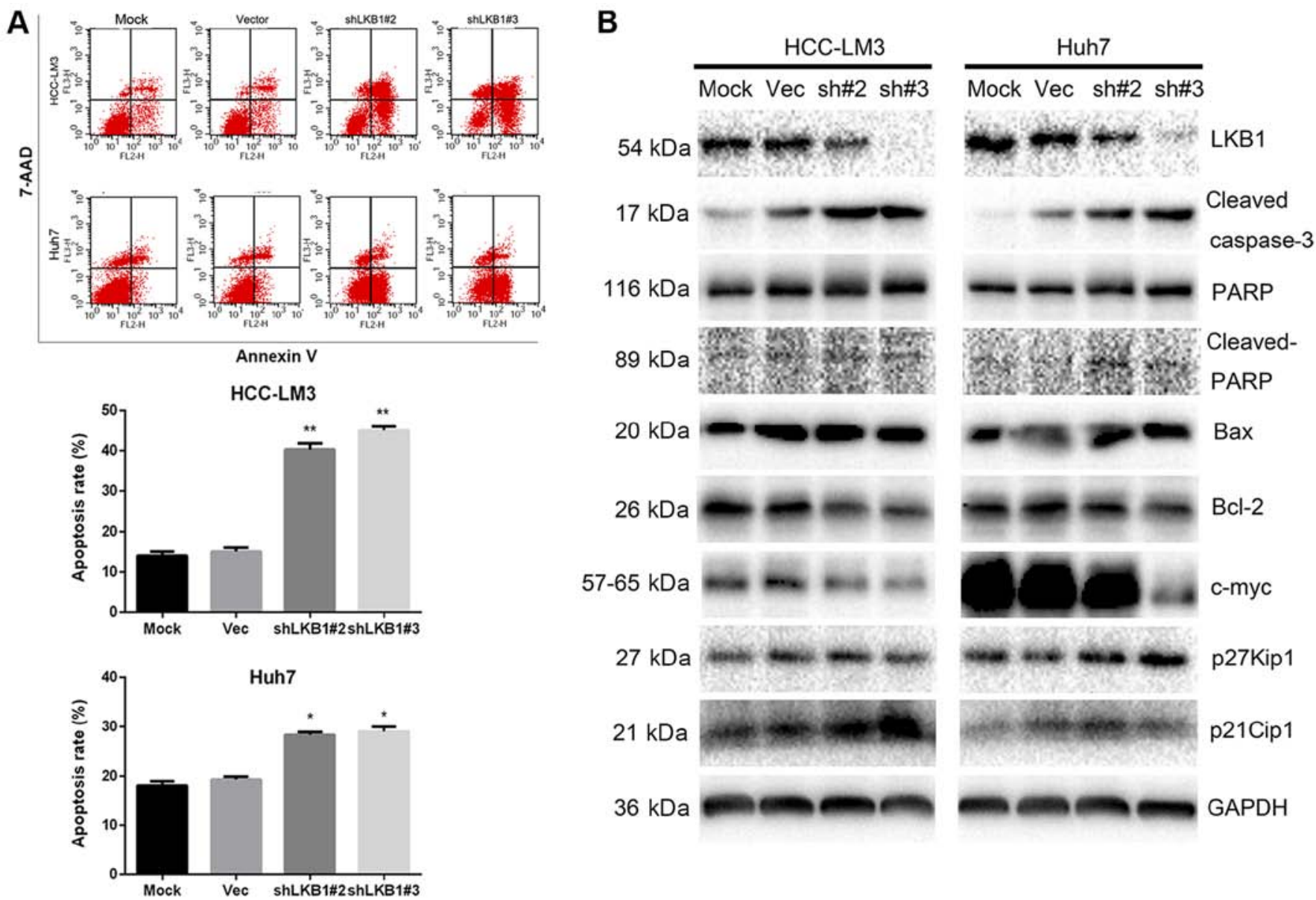

Figure 6. LKB1 knockdown promotes cell apoptosis and regulates proliferation-associated genes. (A) Knockdown of LKB1 promotes cell apoptosis. (B) The expression of cleaved caspase 3 and cleaved PARP was increased as detected by western blot analysis in LKB1-knockdown cells. The expression of the indicated proteins was detected by western blot analysis. ${ }^{* *} \mathrm{P}<0.01,{ }^{*} \mathrm{P}<0.05$. LKB1, liver kinase B1; PARP, poly(adenosine diphosphate ribose) polymerase.

to elucidate the function of LKB1 in different types of cancer. In the present study, the expression pattern of LKB1 was detected in two cohorts of HCC and paired ANT specimens. The results demonstrated that LKB1 was frequently upregulated in HCC tissues, and the high expression of LKB1 was correlated with numerous malignant characteristics, shorter overall survival and earlier recurrence. It was also revealed that a large tumor size, multiple tumor foci, incomplete tumor encapsulation, PVTT, local invasion, vascular invasion, and advanced BCLC or TNM stage were associated with a worse prognosis. Knockdown of LKB1 inhibited cell proliferation by promoting apoptosis and regulating proliferation-associated genes, but overexpression of LKB1 exerted no effect on the proliferation of HCC cells. It is well-known that, under quiescent conditions, LKB1 is localized to the nucleus and activation of LKB1 requires translocation from the nucleus to the cytoplasm by forming a heterotrimer with the proteins STRADA and MO25 $(18,19)$. It may be hypothesized that enhanced LKB1 expression in HCC cells does not affect STRADA and MO25 and, accordingly, LKB1 translocation to the cytoplasm remains unchanged.

HCC is one of the leading causes of cancer-associated mortality worldwide, and its incidence is increasing (20). The Asia-Pacific area is the region with the highest prevalence of $\operatorname{HCC}(21,22)$, and a large number of patients are first diagnosed with $\mathrm{HCC}$ at an advanced stage. Therefore, the therapeutic efficacy is not optimal, and mortality due to cancer recurrence or metastasis is common. In the present study, the protooncogenic role of LKB1 in HCC was demonstrated. Whether and how LKB1 affects HCC metastasis, and the possible therapeutic approaches based on LKB1, remain to be further investigated in future studies.

Germline mutation of LKB1 is responsible for a precancerous condition referred to as Peutz-Jeghers syndrome, which is characterized by the development of benign hamartomatous polyps in the gastrointestinal tract and hyperpigmented macules on the lips and oral mucosa. Patients with Peutz-Jeghers syndrome develop gastrointestinal hamartomas and have a markedly increased risk for developing gastrointestinal, breast and gynecological cancers (23). Dahmani et al (24) reported a novel LKB1 isoform, which lacks the N-terminal region and a portion of the kinase domain, named $\Delta \mathrm{N}$-LKB1. This enhances the metabolic activity of AMPK in HeLa cells and NCI-H460 lung cancer cells and has intrinsic oncogenic properties. In order to explore the possibility of mutated LKB1 in HCC tissues and cell lines used in the present study, the literature on LKB1 mutation in HCC was reviewed. Kim et al (25) collected 80 HCC samples and 7 dysplastic nodules to investigate potential mutations in all 9 exons of LKB1. The results revealed the presence of only one missense mutation of $\mathrm{CCG} \rightarrow \mathrm{CTG}(\mathrm{Pro} \rightarrow \mathrm{Leu})$ 
among the $80 \mathrm{HCC}$ cases, whereas no mutation was identified among the 7 dysplastic nodules. Pineau et al (26) collected 57 hepatobiliary cancer cell lines for detection of homozygeous deletions, and no homozygous deletion of LKB1 was detected in the HCC cell lines used in their study. Therefore, the effect of LKB1 observed in the present study was likely exerted by a non-mutated protein.

Activation of LKB1 by phosphorylation at the Ser428, Ser307 and Ser399 sites is required for translocation from the nucleus to the cytoplasm (27-29). It has been reported that LKB1 regulates glucose metabolism and suppresses gluconeogenesis in the normal liver (30), and knockout of LKB1 in mouse livers leads to the inability to use glucose, resulting in severe hyperglycemia (31). Apoptosis is a type of programmed cell death under various types of stress $(32,33)$. It is reasonable to hypothesize that LKB1-knockdown cells underwent apoptosis due to inability to use glucose. LKB1 may be used as a potential therapeutic target in $\mathrm{HCC}$ treatment by agents suppressing phosphorylation at Ser428, Ser307 and Ser399, thereby inhibiting nuclear export of LKB1.

The in vivo tumor inhibitory effect of LKB1 was previously investigated by knockout of LKB1 in mice $(34,35)$, and the most recent study indicated that LKB1 acts as a master gatekeeper of liver regeneration (36). Another previous study indicated that LKB1 was downregulated in HCC and that low expression is correlated with poor prognosis (10). This conclusion was made based on IHC staining analysis of 70 cases. In the present study, in which the scale of samples included was enlarged, different conclusions were reached. Along with the results of previous studies $(8,9,37)$, the present study suggests that LKB1 plays a proto-oncogenic role in HCC. It is suggested that the function of LKB1 varies between different cancer types and pathological conditions. Therefore, the heterogeneity of cancers should be taken into consideration in cancer therapy.

\section{Acknowledgements}

The authors would like to thank Changshu Ke and Jing Xiong (Department of Pathology, Tongji Hospital) for their assistance with the IHC scoring, Zhanguo Zhang (Hepatic Surgery Center, Tongji Hospital) for their assistance with the shRNA lentivirus production, Xiaolan Li (Public Experimental Platform, Tongji Hospital) for conducting the flow cytometry, Lanping Ding (Institute of Organ Transplantation, Tongji Hospital) and Shunchang Zhou (Department of Experimental Zoology, Tongji Medical College) for animal care, and Wei Dong (Hepatic Surgery Center, Tongji Hospital) for insightful discussion.

\section{Funding}

The present study was supported by the National Natural Science Fund (grant nos. 31671348, 81572427 and 81572855).

\section{Availability of data and materials}

All data generated or analyzed during the present study are included in this published article. The authors declare that materials described in the manuscript, including all relevant raw data, will be freely available to any scientist wishing to use them for non-commercial purposes, without breaching participant confidentiality.

\section{Authors' contributions}

XT and LC designed the experiments, XT and ZL performed the experiments. ZL collected clinicopathological data. XT, BZ, XC and LC analyzed the results. XT and ZL generated the data, prepared the panels and assembled the figures and tables. XT and LC wrote the manuscript. All authors have reviewed and approved the final version of the manuscript.

\section{Ethics approval and consent to participate}

The protocol of the present study, involving both human clinical samples and animal experimentation, was approved by the Ethics Committee of Tongji Hospital, Huazhong University of Science and Technology. Written informed consent was obtained from all patients.

\section{Patient consent for publication}

Not applicable.

\section{Competing interests}

The authors declare that they have no competing interests.

\section{References}

1. Alessi DR, Sakamoto K and Bayascas JR: LKB1-dependent signaling pathways. Annu Rev Biochem 75: 137-163, 2006.

2. Hardie DG: AMP-activated/SNF1 protein kinases: Conserved guardians of cellular energy. Nat Rev Mol Cell Biol 8: 774-785, 2007.

3. Liu W, Monahan KB, Pfefferle AD, Shimamura T, Sorrentino J, Chan KT, Roadcap DW, Ollila DW, Thomas NE, CastrillonDH, et al: LKB1/STK11 inactivation leads to expansion of a prometastatic tumor subpopulation in melanoma. Cancer Cell 21: 751-764, 2012.

4. Li F, Han X, Li F, Wang R, Wang H, Gao Y, Wang X, Fang Z, Zhang $\mathrm{W}$, Yao $\mathrm{S}$, et al: LKB1 Inactivation elicits a redox imbalance to modulate non-small cell lung cancer plasticity and therapeutic response. Cancer Cell 27: 698-711, 2015.

5. Herrmann JL, Byekova Y, Elmets CA and Athar M: Liver kinase B1 (LKB1) in the pathogenesis of epithelial cancers. Cancer Lett 306: 1-9, 2011.

6. Bardeesy N, Sinha M, Hezel AF, Signoretti S, Hathaway NA, Sharpless NE, Loda M, Carrasco DR and DePinho RA: Loss of the Lkb1 tumour suppressor provokes intestinal polyposis but resistance to transformation. Nature 419: 162-167, 2002.

7. Jeon SM, Chandel NS and Hay N: AMPK regulates NADPH homeostasis to promote tumour cell survival during energy stress. Nature 485: 661-665, 2012.

8. Martinez-Lopez N, Garcia-Rodriguez JL, Varela-Rey M, Gutiérrez V, Fernández-Ramos D, Beraza N, Aransay AM, Schlangen K, Lozano JJ, Aspichueta $\mathrm{P}$, et al: Hepatoma cells from mice deficient in glycine $\mathrm{N}$-methyltransferase have increased RAS signaling and activation of liver kinase B1. Gastroenterology 143: 787-798.e13, 2012.

9. Lee SW, Li CF, Jin G, Cai Z, Han F, Chan CH, Yang WL, Li BK, Rezaeian AH, Li HY, et al: Skp2-dependent ubiquitination and activation of LKB1 is essential for cancer cell survival under energy stress. Mol Cell 57: 1022-1033, 2015.

10. Huang Y-H, Chen Z-K, Huang K-T, Li P, He B, Guo X, Zhong JQ, Zhang QY, Shi HQ, Song QT, et al: Decreased expression of LKB1 correlates with poor prognosis in hepatocellular carcinoma patients undergoing hepatectomy. Asian Pac J Cancer Prev 14: 1985-1988, 2013. 
11. Bruix J and Sherman M; American Association for the Study of Liver Diseases: Management of hepatocellular carcinoma: An update. Hepatology 53: 1020-1022, 2011.

12. Li JC, Yang XR, Sun HX, Xu Y, Zhou J, Qiu SJ, Ke AW, Cui YH, Wang ZJ, Wang WM, et al: Up-regulation of Kruppel-like factor 8 promotes tumor invasion and indicates poor prognosis for hepatocellular carcinoma. Gastroenterology 139: 2146-2157.e12, 2010.

13. Edmondson HA and Steiner PE: Primary carcinoma of the liver: A study of 100 cases among 48,900 necropsies. Cancer 7: 462-503, 1954

14. Zhou L, Rui J-A, Ye D-X, Wang S-B, Chen S-G and Qu Q: Edmondson-Steiner grading increases the predictive efficiency of TNM staging for long-term survival of patients with hepatocellular carcinoma after curative resection. World J Surg 32: 1748-1756, 2008.

15. Moffat J, Grueneberg DA, Yang X, Kim SY, Kloepfer AM, Hinkle G, Piqani B, Eisenhaure TM, Luo B, Grenier JK, et al: A lentiviral RNAi library for human and mouse genes applied to an arrayed viral high-content screen. Cell 124: 1283-1298, 2006

16. Zhang B, Halder SK, Kashikar ND, Cho YJ, Datta A, Gorden DL and Datta PK: Antimetastatic role of Smad4 signaling in colorectal cancer. Gastroenterology 138: 969-980.e1-3, 2010.

17. Han D, Li SJ, Zhu YT, Liu L and Li MX: LKB1/AMPK/mTOR signaling pathway in non-small-cell lung cancer. Asian Pac J Cancer Prev 14: 4033-4039, 2013.

18. Baas AF, Boudeau J, Sapkota GP, Smit L, Medema R, Morrice NA, Alessi DR and Clevers HC: Activation of the tumour suppressor kinase LKB1 by the STE20-like pseudokinase STRAD. EMBO J 22: 3062-3072, 2003.

19. Boudeau J, Baas AF, Deak M, Morrice NA, Kieloch A, Schutkowski M, Prescott AR, Clevers HC and Alessi DR: MO25alpha/beta interact with STRADalpha/beta enhancing their ability to bind, activate and localize LKB1 in the cytoplasm. EMBO J 22: 5102-5114, 2003.

20. Siegel RL, Miller KD and Jemal A: Cancer statistics, 2016. CA Cancer J Clin 66: 7-30, 2016

21. Ferlay J, Soerjomataram I, Dikshit R, Eser S, Mathers C, Rebelo M, Parkin DM, Forman D and Bray F: Cancer incidence and mortality worldwide: Sources, methods and major patterns in GLOBOCAN 2012. Int J Cancer 136: E359-E386, 2015.

22. Torre LA, Bray F, Siegel RL, Ferlay J, Lortet-Tieulent J and Jemal A: Global cancer statistics, 2012. CA Cancer J Clin 65: 87-108, 2015

23. Korsse SE, Peppelenbosch MP and van Veelen W: Targeting LKB1 signaling in cancer. Biochim Biophys Acta 1835: 194-210, 2013.

24. Dahmani R, Just PA, Delay A, Canal F, Finzi L, Prip-Buus C, Lambert M, Sujobert P, Buchet-Poyau K, Miller E, et al: A novel LKB1 isoform enhances AMPK metabolic activity and displays oncogenic properties. Oncogene 34: 2337-2346, 2015.

25. Kim CJ, Cho YG, Park JY, Kim TY, Lee JH, Kim HS, Lee JW, Song YH, Nam SW, Lee SH, et al: Genetic analysis of the LKB1/ STK11 gene in hepatocellular carcinomas. Eur J Cancer 40: $136-141,2004$
26. Pineau P, Marchio A, Nagamori S, Seki S, Tiollais P and Dejean A: Homozygous deletion scanning in hepatobiliary tumor cell lines reveals alternative pathways for liver carcinogenesis. Hepatology 37: 852-861, 2003.

27. Xie Z, Dong Y, Scholz R, Neumann D and Zou MH: Phosphorylation of LKB1 at serine 428 by protein kinase C-zeta is required for metformin-enhanced activation of the AMP-activated protein kinase in endothelial cells. Circulation 117: 952-962, 2008.

28. Xie Z, Dong Y, Zhang J, Scholz R, Neumann D and Zou MH: Identification of the serine 307 of LKB1 as a novel phosphorylation site essential for its nucleocytoplasmic transport and endothelial cell angiogenesis. Mol Cell Biol 29: 3582-3596, 2009.

29. Zhu H, Moriasi CM, Zhang M, Zhao Y and Zou MH: Phosphorylation of serine 399 in LKB1 protein short form by protein kinase $\mathrm{C} \zeta$ is required for its nucleocytoplasmic transport and consequent AMP-activated protein kinase (AMPK) activation. J Biol Chem 288: 16495-16505, 2013.

30. Patel K, Foretz M, Marion A, Campbell DG, Gourlay R, Boudaba N, Tournier E, Titchenell P, Peggie M, Deak M, et al: The LKB1-salt-inducible kinase pathway functions as a key gluconeogenic suppressor in the liver. Nat Commun 5: 4535, 2014.

31. Shaw RJ, Lamia KA, Vasquez D, Koo SH, Bardeesy N, Depinho RA, Montminy M and Cantley LC: The kinase LKB1 mediates glucose homeostasis in liver and therapeutic effects of metformin. Science 310: 1642-1646, 2005.

32. Evan G and Littlewood T: A matter of life and cell death. Science 281: 1317-1322, 1998.

33. Lowe SW, Cepero E and Evan G: Intrinsic tumour suppression. Nature 432: 307-315, 2004.

34. Miyoshi H, Deguchi A, Nakau M, Kojima Y, Mori A, Oshima M, Aoki M and Taketo MM: Hepatocellular carcinoma development induced by conditional beta-catenin activation in $\mathrm{Lkb1}^{+/-}$mice. Cancer Sci 100: 2046-2053, 2009.

35. Nakau M, Miyoshi H, Seldin MF, Imamura M, Oshima M and Taketo MM: Hepatocellular carcinoma caused by loss of heterozygosity in Lkb1 gene knockout mice. Cancer Res 62: 4549-4553, 2002.

36. Maillet V, Boussetta N, Leclerc J, Fauveau V, Foretz M, Viollet B, Couty JP, Celton-Morizur S, Perret C and Desdouets C: LKB1 as a gatekeeper of hepatocyte proliferation and genomic integrity during liver regeneration. Cell Reports 22: 1994-2005, 2018.

37. Martínez-López N, Varela-Rey M, Fernández-Ramos D, Woodhoo A, Vázquez-Chantada M,EmbadeN,Espinosa-Hevia L, Bustamante FJ, Parada LA, Rodriguez MS, et al: Activation of LKB1-Akt pathway independent of phosphoinositide 3-kinase plays a critical role in the proliferation of hepatocellular carcinoma from nonalcoholic steatohepatitis. Hepatology 52: $1621-1631,2010$

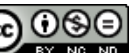

This work is licensed under a Creative Commons Attribution-NonCommercial-NoDerivatives 4.0 International (CC BY-NC-ND 4.0) License. 